\title{
Challenging behaviour in dementia care: a novel framework for translating knowledge to practice
}

\author{
Ian A. James ${ }^{1, *}$, Katharina Reichelt ${ }^{1}$, Esme Moniz-Cook ${ }^{2}$ and Katy Lee ${ }^{3}$ \\ ${ }^{1}$ Newcastle Department of Older Adult Clinical Psychology, Akenside Offices, Cumbria, Northumberland, Tyne and Wear \\ NHS Foundation Trust, Westgate Road, Newcastle upon Tyne NE4 6BE, UK, ${ }^{2}$ Faculty of Health Sciences, Dearne Building \\ Room 109, University of Hull, Hull HU6 7RX, UK and ${ }^{3}$ Intensive Support Service for Older People, Surrey and Borders \\ Partnership NHS Foundation Trust, UK \\ *Corresponding author. Email: Ianandrew.james@cntw.nhs.uk
}

(Received 31 March 2020; revised 3 July 2020; accepted 12 August 2020)

\begin{abstract}
This article provides guidance on the management of challenging behaviours (CBs) in dementia care, and introduces concepts from positive behavioural support not usually applied to dementia. While the use of formulations has received a lot of attention in recent years, the mechanisms of how to apply the formulation-led interventions requires more consideration. In order to assist caregivers to deliver effective interventions we need to have a better understanding of the CBs we are attempting to manage, and also produce management strategies with clearer goals. Ideally we would also want caregivers to be able to describe the skills they employ in a coherent manner in order to facilitate selfreflection and to be able to pass on their skills to junior colleagues. This paper attempts to fulfil these needs by integrating two new models with philosophies already used in dementia care. In terms of new concepts, the first is the Arousal Cycle, which gives caregivers an awareness of the five phases of a typical $\mathrm{CB}$ (wellbeing, trigger, escalation, $\mathrm{CB}$, and recovery phase). In relation to the second, the Traffic Light analogy examines $\mathrm{CBs}$ in terms of four management stages: primary prevention, secondary prevention, reactive strategies, and calming strategies. It is proposed that we distinguish between these stages when composing our formulations and care plans, and thereby produce better targeted interventions. By the end of the paper the reader will have been presented with material integrating concepts from the fields of dementia and intellectual impairment, and been introduced to new ways of managing CBs.
\end{abstract}

\section{Key learning aims}

After reading this article people will:

(1) Be provided with more specific guidance regarding the management of challenging behaviour (CB) in dementia; such guidance was not provided by the update of the NICE guidelines for dementia (2018).

(2) Appreciate that the unmet needs perspective helps us both to understand why CB occur and to select appropriate management strategies.

(3) Have an increased awareness and knowledge of new models from outside of the field of dementia. For example, through the use of the 'arousal cycle' people can recognise that a CB should more realistically be seen as having different phases (beginning, middle, end) rather than being perceived as a single action.

(4) Be introduced to the traffic light conceptualisation which provides a useful way for guiding management strategies.

(5) Be aware of when best to use resource-intensive formulations.

(6) Recognise that in addition to conceptualising the person in relation to the $\mathrm{CB}$, it is helpful to conceptualise the structural elements of the behaviour too. 
(7) Appreciate the need to help caregivers to recognise their existing skills, and to give carers the means to be able to articulate these abilities. Many care home staff currently work intuitively in the way they deliver care; as such we think they require practical frameworks and protocols to help them better elucidate what they do.

Keywords: conceptualisation; formulation; NICE; positive behavioural support; training

\section{Introduction to challenging behaviours}

In the UK the most recent NICE (2018) guidelines for the treatment of non-cognitive symptoms (the NICE term used for challenging behaviour) state it is important to: explore possible reasons for people's distress; check for and address clinical or environmental causes (e.g. pain, delirium or inappropriate care); and offer personalised activities to promote engagement, pleasure and interest. While these suggestions are useful, they fail to give any specific guidance about what to do in this important clinical area. Other reviews present lists of potential treatments, often noting a poor and inconsistent evidence base, but also failing to make clear recommendations about treatment approaches (Rapaport et al., 2017). It is in this context of overly generic and unspecific recommendations that we produce the following article. It provides theory-led recommendations for the management of challenging behaviours (CBs).

\section{Behaviours}

Cohen-Mansfield (2000) distinguishes between CBs in terms of agitated, non-agitated and inactive (depressed/apathy based) presentations. Our paper is concerned with behaviours that are disruptive to others and therefore excludes conditions described as apathy and depression. We do this to side-step the controversy to do with whether low mood and apathy are linked directly to the type of cognitive impairments commonly found in dementia (Cerejeira et al., 2012) which is outside the scope of this paper. Non-agitated behaviours (excessive walking, refusing to get out of bed, repetitive questioning, refusal of essential care/medication) may ultimately require intervention to maintain the wellbeing of the person or others, and thus can lead to confrontation. Therefore on occasions when staff have to intervene with a nonagitated $\mathrm{CB}$, or it is left unmanaged, this can turn into an agitated behavioural type. Agitated CBs (shouting, throwing items, kicking doors, pulling hair) often result in confrontation, requiring caregivers to de-escalate the situation.

There are numerous causes for the behaviours: physical, environmental, social, psychological and drug-induced. Establishing and dealing with the cause of the behaviours may reduce or stop the $\mathrm{CB}$ from occurring in the present or future. In all circumstances, however, when a problematic behaviour arises, the caregivers need to intervene and communicate in a way that reduces distress and the emotionality of the situation, while also supporting the person with dementia (McCallion et al., 1999). Indeed, even when the cause of the $\mathrm{CB}$ is physical in origin (acute pain, delirium), the caregiver's response to the person's distress will either increase or decrease the likelihood of an escalation of any problematic behaviour.

\section{Causes}

Identifying the cause of a $\mathrm{CB}$ is important in relation to devising a care plan (treatment plan or behavioural support plan). Frequently, there are multiple factors directly and indirectly influencing the actions of the person with dementia $(\mathrm{PwD})$, rather than a single cause. Cunningham et al. (2019) suggest each $\mathrm{CB}$ has at least four contributing factors, the two most common being pain and negative caregiver interactions. People's physical health, mental health and cognitive status interact and determine how they navigate their way around their environment. The environment (noise, lighting, layout, etc.) will either help or hinder their 
Table 1. Common causes of $\mathrm{CB}$

\begin{tabular}{ll}
\hline Physical health issues & $\begin{array}{c}\text { Pain, dental pain; delirium; infections; constipation; thyroid functioning; diabetes; } \\
\text { brain metastases } \\
\text { Mental health issues }\end{array}$ \\
$\begin{array}{c}\text { Psychosis; paranoia; depression; anxiety (panic, social phobia, PTSD, etc.); autism; } \\
\text { loneliness; trauma } \\
\text { Environmental issues }\end{array}$ & $\begin{array}{r}\text { Poor layout of the environment; over/under stimulation; setting felt as being overly } \\
\text { restrictive; physical discomfort due to temperature, noise, smells, furniture and fixings, } \\
\text { including lighting } \\
\text { Sense of isolation and loneliness; lack of a sense of belonging; verbal and non-verbal } \\
\text { behaviour of other people in the environment, including caregivers }\end{array}$ \\
\hline
\end{tabular}

wellbeing and coping skills in that setting. Some of the common causes associated with CBs across the relevant domains are listed below (Table 1).

It is important to remember that some of the above causes are routinely treated with psychotropic medication. However, this can also produce unwanted side-effects: e.g. increasing levels of confusion, tiredness, agitation, constipation and, in the case of some medications for Parkinson's disease, hyper-sexuality (Banerjee, 2009). Owing to the severity of the side-effects for many people with dementia, there has been a national programme to reduce the prescribing rates of anti-pychotics, which has achieved partial success (HSJ, 2020).

\section{Philosophies used in the management of $\mathrm{CB}$}

We suggest that there are universal notions or philosophies that apply to the management of CB; we will examine these features prior to introducing new concepts from outside of the traditional dementia literature. All CBs should be understood and managed using these notions. Our three key philosophies are: (i) the use of the 'unmet needs perspective'; (ii) the relevance of good communication skills in delivering effective care; and (iii) the use of enhanced care-planning to deal with risky or persistent $\mathrm{CB}$ via the use of formulations.

The 'unmet needs perspective' has been described well by Algase et al. (1996) and Cohen-Mansfield (2000). They proposed that CBs are an expression of distress that arises from physical or psychological unmet needs. For example, Cohen-Mansfield (2000) suggests that behaviours often reflect an attempt by a person to signal a need that is currently not being met (e.g. to indicate hunger, to gain relief from pain or boredom, etc.), an effort by an individual to get his needs met directly (e.g. leave a building when he believes he must either go to work or collect children from school) or a sign of frustration (e.g. feeling angry at being told he is not allowed to exit a building). In all of these situations, the actions are attempts by the individual to enhance and maintain his/her sense of well-being or to ease distress. Therefore identifying and resolving the unmet needs should be the focus of treatment. Despite the widespread use of the concept of 'need' in the field of dementia care, it suffers from not having a clear consensus about its meaning. In an attempt to achieve some clarity, James and Jackman (2017) produced an 8-item needs framework made up of the following eight needs: physical comfort, perception of safety, positive touch, love and belonging, self-esteem, control over environment and possessions, fun, and occupation and exploration. The needs framework has been useful when using a checklist to mark off which needs may be going unmet within a setting where a PwD is displaying agitation (James and Reichelt, 2019).

The second philosophy concerns the importance of caregivers being able to communicate and interact effectively with PwD. In the field of dementia the agent of change is usually the caregiver rather than the person with dementia, hence effective carer communications skills are essential. Good communication will reduce levels of stress and distress, while poor communication may increase illbeing, thereby leading people to feel unsafe, overly controlled or disrespected. It is interesting to note that, while caregivers are able to identify good examples of communication 
skills, they are poorer at articulating what constitutes good communication. Empirical evidence (Rapaport et al., 2017) and clinical observations suggest that such self-awareness is crucial, because it informs caregivers why something 'went well', or 'why it didn't'. In recent years the authors have developed a training programme in this area called CAIT (Communication And Interaction Training; James and Gibbons, 2019). This programme incorporates the Newcastle model, and helps caregivers to identify their strengths and weaknesses in relation to current communication skills.

The final philosophy concerns the use of formulations. Formulations have received a great deal of attention in recent years For example, Holle et al. (2016) undertook an extensive review of different types of formulations used in the management of CB; they identified 14 biopsychosocial models which they examined in detail. Moniz-Cook et al. (2012) reviewed treatments used in $\mathrm{CB}$ from a functional analytic perspective for Cochrane. In complex presentations the use of formulations is highly recommended (James and Moniz-Cook, 2018), and they should be tailored to the individual's needs, providing relevant information to develop a comprehensive care plan. It is important to note, however, that because CBs are so frequent, occurring hourly or more, across a range of care settings, it is impractical to suggest formulations are used with all CBs (James and Gibbons, 2019; James and Reichelt, 2019). Brechin et al. (2013) have produced a stepped-care treatment algorithm, starting from 'watchful waiting' to 'the use of formulations' at the top of the hierarchy. One of the current difficulties in relation to the use of formulations is that not all clinical teams or organisations have the confidence, knowledge or skills to use them. This is because many of the caregivers who could potentially be involved in formulating (e.g. care home staff from privately run homes) do not have a background or training in mental health; so the term 'formulation' itself is ' . . o off-putting' to some caregivers (James and Reichelt, 2019). A further issue is the fact that to date insufficient attention has been paid to how the products of the formulations and care plans are being implemented. Indeed, the value of the formulation is undermined if the resulting recommendations are poorly implemented due to a lack of understanding, insufficient training or poor skills of the caregivers. This article attempts to address some of these issues by presenting helpful models and clearer practical guidance.

\section{Understanding the structural phases of CBs}

In the dementia literature CBs have tended to be treated as singular events, and the idea they are composed of discrete phases is novel. However, following a brief reflection one can accept they must be composed of discrete phases and there is an advantage in treating them as such. A review of the literature suggests that the phases are best described in terms of the 'Arousal Cycle Graph'. This graph describes five phases: wellbeing, trigger, escalation, CB and recovery (see Fig. 1, adapted from Kaplan and Wheeler, 1983; Hallet, 2018). Identifying the phase the person is 'in' will guide one's interventions. During the wellbeing phase the person is neither stressed nor distressed. The active phases of the graph begin with a trigger that initiates the person's agitation. During the trigger phase, it is still possible for others to calm the person, or for the person to calm themselves. In the escalation phase, the person's agitation grows and their agitation increases. As the individual's arousal increases, this affects their ability to think as hypothesised in compassionate focused therapy theory (Gilbert, 2014). People with dementia are in double jeopardy at this stage because their thinking is being negatively influenced by both the cognitive deficits associated with the dementia and their frontal lobes being overwhelmed due to the stressful situation. The CB phase occurs when the person's arousal levels continue to rise. During this phase, it will be increasingly difficult for the person to respond to standard social interactions, accept different perspectives or be reasoned with. They will be less likely to respond to simple distraction techniques, and caregivers may sometimes have to adopt a physical response, for example escape, self-defence or restraint 


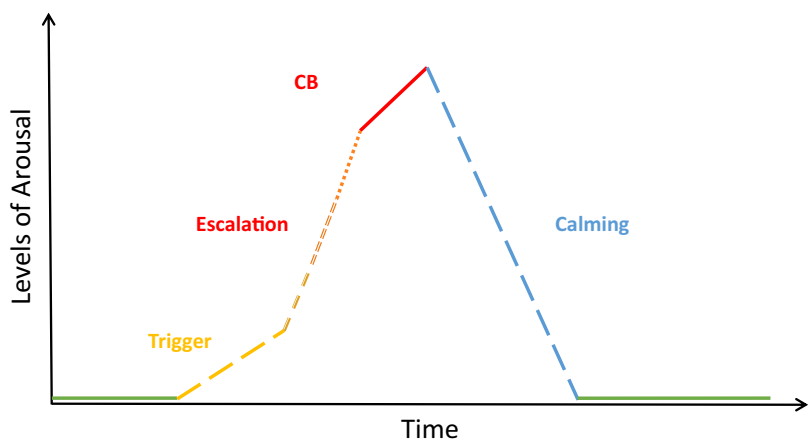

Figure 1. Adapted version of the Arousal Cycle Graph.

of the individual. In this phase, the main aim of those attending to the situation is to ensure the safety of the individual, themselves and others involved. It is also imperative that the caregivers remain calm, display an open body language and contain strong emotions. As the person's agitation subsides, they enter the calming recovery phase, and gradually return to their baseline mood. Although the immediate episode may have passed, the person's anger can easily be re-triggered, particularly early on. Figure 1 clarifies that a $\mathrm{CB}$ is a sequence of events, and by intervening at different points of this sequence, escalation may be averted.

The Traffic Light analogy is a clinically useful analogy based on the same concepts as the Arousal Cycle Graph. It has been used for a number of years in Positive Behavioural Support programmes for the treatment of CBs in people with learning disabilities (Hallet, 2018). Its use has been adapted for working with people with dementia by Sells and Shirley (2010) in a helpful illustrative article. The analogy uses the three colour sequences of a traffic light (green, amber, red), plus a blue phase; this is described briefly below, and its clinical utility is outlined more comprehensively later in the article.

Primary prevention 'green' strategies aim to support the person with dementia to be content and calm, and is associated with the wellbeing phase of the arousal cycle. Such approaches should be designed to meet a person's needs and foster safe and stimulating environments. Secondary prevention 'amber' strategies guide responses to the early warning signs, to help caregivers intervene timely and appropriately when the first signs of stress are observed. Amber is linked with the 'trigger' and early escalation phases of the graph, and here we see numerous examples of de-escalation strategies. Reactive 'red' strategies aim to manage behaviours safely and quickly once the $\mathrm{CB}$ has been triggered. A mixture of de-escalation and physical techniques may be required here, with restraining methods being the approach of last resort. Calming 'blue' strategies (post-incident support) aim to prevent re-triggering of the $\mathrm{CB}$, and learning relevant lessons from the episode. In the remainder of the article, we discuss management and treatment strategies based on the ideas and models discussed above.

\section{Management and treatments}

In this section the above theories and models are used to discuss 'what to do' with respect to the management of CB. The first set of strategies is linked to the traffic light model, and corresponds closely to the phases of the arousal cycle. Secondly, we review the clinical implications of the models.

\section{Management approaches linked to the phases and traffic light analogy}

Many of the previous guidelines of the treatment of CB have been rather vague, and this includes the recent NICE (2018) guidelines. The traffic light conceptualisation and associated phases provides clearer guidance on what methods and actions to employ with CB. 
Box 1. Examples of primary prevention

\begin{abstract}
John - this fiercely independent 85-year-old man valued his freedom, and he loved doing projects around the house. When he went into a care home he found the rules and structure very restricting. 'Meeting his needs' involved giving him the opportunity to do art/craft projects, and work in the care home's large garden.

Mary - a sociable 78-year-old loved the company of others. Owing to her mobility problems she needed help to visit friends and attend church. Her needs were best met by giving her access to chats and social gatherings, and the ability to stream live church services on a computer.
\end{abstract}

\title{
Primary prevention (green zone)
}

Primary prevention aims to prevent $\mathrm{CBs}$ from occurring in the first place, maintaining people in a state of wellbeing. Table 1 describes some of the common triggers of challenging behaviours; by addressing these we may be able to avoid a CB being triggered in the first place. Also, using our unmet needs perspective, we can aim to identify people's fundamental needs and ensure they are being met. Such needs include: being free from discomfort and pain; feeling respected; appropriately occupied, etc. It should be acknowledged, however, that because individuals have different priorities and preferences, a degree of flexibility and tailoring will be required. Hence individualised care planning is vital in the delivery of a needs-led intervention, as well as considering how to adapt valued roles or activities to the person's current abilities (Box 1).

Primary prevention requires bespoke care planning. PwD should be involved in care planning where possible, and family members and carers may also provide information on potential triggers as well as patterns of behaviour (NHS Protect, 2017).

Figure 2 shows that most of the time PwD are not actively 'challenging'. The aim of good primary prevention is to ensure that both the number and the frequency of 'red lights' are low, and time spent between 'red signals' is long. Therefore, we recommend the use of good person-centred approaches as described in projects like WHELD (Ballard et al., 2016). We think it is essential that we promote programmes that have clearly articulated the mechanisms by which person-centred care is achieved (Dementia Care Mapping, VIPS; Brooker and Latham, 2015).

Dementia Care Mapping (DCM; Kitwood, 1997) was designed to empower staff teams to improve the quality of care for $\mathrm{PwD}$. It was primarily developed as part of a quality improvement process to enhance the quality of person-centred care over time, and thus is an excellent example for primary prevention. Through a process of observation and feedback, staff are encouraged to consider care from the point of view of the person living with dementia. On the basis of these observations, changes are made to care plans and to clinical practice.

The 'VIPS' framework (Brooker and Latham, 2015) sums up the elements in Kitwood's philosophy of person centred care for persons with dementia as: values, individualised approach, the perspective of the person living with dementia and the social environment. In this evidence-based approach there are six indicators for each VIP element. The framework provides helpful practical guidance on whether person-centred care is being delivered and how it can be enhanced.

The other types of treatment strategies to be used in primary prevention are the non-pharmacological approaches, such as CST (cognitive stimulation therapy) which has a robust evidence base. In contrast, the evidence for other methods (aromatherapy, music therapy, psychomotor) is relatively poor and efficacy rather inconsistent (Livingston et al., 2014). We feel there are a number of reasons for this, including (i) a lack of agreement about what constitutes a particular therapy; and (ii) difficulties with measuring appropriate change. 


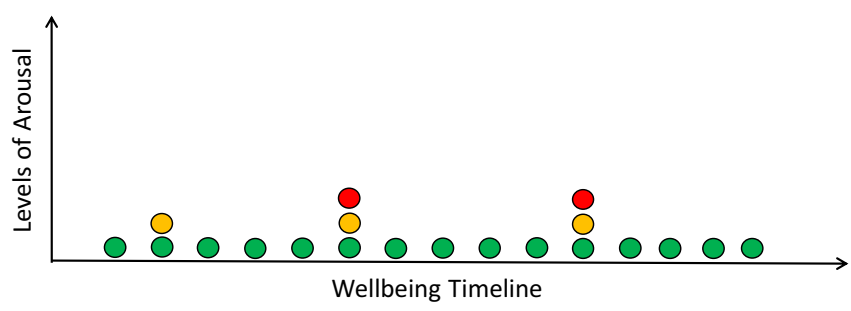

Figure 2. Traffic light analogy timeline.

For example, 'music therapy' in one setting is often different from a similarly labelled therapy in another setting. This is the case in most of the non-pharmacological approaches, apart from CST. The clear format provided in the CST manual allows one to examine adherence and dosage of therapy. Apart from CST, the studies in this area are often unclear or unrealistic about what the change mechanisms are in relation to the therapy, and thus are often measuring inappropriate signs and symptoms of efficacy. A clearer understanding of how each therapy impacts mood, wellbeing, behavioural change, etc. is required (Rapaport et al., 2017; Bunn and Handley, 2019). James and colleagues (James and Jackman, 2017; James and Reichelt, 2019) have been working on this issue for some time, and reconceptualised many of the nonpharmacological approaches as 'vehicles' for meeting people's needs; with the hypothesis that the more needs being met within the intervention the more effective the intervention. In support of this reframing the authors developed the Needs Checklist to assess whether people's needs are being met during an intervention. Using this perspective the specific type of therapy (CST, music) is less important, rather caregivers should be asking themselves whether they are meeting people's needs, and also can the intervention be improved by targeting more needs.

\section{Secondary prevention (amber zone)}

Unfortunately, caregivers are not always able to meet people's needs, and many of the causes of CB relate to this inability. If healthcare staff are aware of potential triggers in their clinical area, they might be able to intervene before triggers become an issue. However, where this is not possible, being aware of triggers can enable caregivers to respond empathetically. Thus it is often useful to get staff to think about two different types of triggers to CBs: predictable and unpredictable. Predictable triggers may include 'intimate care interventions', particular 'people' or 'times of the day' that are probably going to distress an individual (e.g. noisy environment, a personal care intervention). Staff are usually good at specifying these situations and may already have developed strategies for dealing with them. In terms of unpredictable triggers, staff are not currently aware that there are triggers to the behaviours and therefore may be ill prepared to deal with the consequences. Drawing on Positive Behaviour Support theory, many unpredictable triggers reflect what are known as 'slow' triggers in this field. Slow triggers are factors that may have a delayed impact on a person's behaviour, which can include boredom, over/under-stimulation, physical discomfort, tiredness or hunger.

To assist with the less predictable CBs we encourage staff to look for patterns and try to identify more subtle triggering factors, so that staff can be better prepared and feel more in control of the situation. To improve the predictability the use of $\mathrm{ABC}$ charts are important, and also the development of formulation frameworks. One of the advantages of using monitoring charts and formulations is that the caregivers become more observant of the people with dementia, and are better able to recognise changes in their presentations (signs of wellbeing and distress) owing to the greater level of general observations.

Once the CB has been triggered the caregiver often needs to take action to deal with it before it escalates to a crisis point. Hence, an awareness and confidence in the use of de-escalation strategies is 
essential. In such circumstances we would advocate techniques to reduce the energy in the situation and then seek ways to meet the person's needs. Several de-escalation approaches have been outlined by James and Gibbons (2019), including BANGs (Macaulay, 2015), environmental manipulation and therapeutic lying (James, 2015). A comprehensive review of the common de-escalation strategies in the UK has been produced recently by James, Medea and Reichelt (in press).

\section{Reactive strategies (red zone)}

These correspond to the 'CB phase' of the arousal cycle. While all the previous techniques are relevant at this stage (i.e. de-escalation, environmental manipulation, therapeutic lying, etc.), this is the only stage where physical interventions are used. Physical interventions are only employed as a final resort (Howarth et al., 2017). Such approaches involve physical interventions including: manual restraint, which is a 'skilled, hands-on method of physical restraint used to prevent service users from harming themselves, endangering others or compromising the therapeutic environment'. Physical interventions should only be undertaken by people who have received appropriate training (Howarth et al., 2017) due to the risks involved with incorrect application of physical interventions, which include mental trauma and physical injury (NHS Protect, 2017). Important work in the area of dementia regarding this topic has been undertaken by members of the Northumberland Behavioural Support Service (Howarth and Sells, 2014) who have produced a formulation-led physical restraint framework. In addition to the use of restrictive practices in the red zone, it is important for the staff supporting the person to model calmness and emotional containment. This is especially true when supporting people with dementia who may become more confused or distressed if lots of staff are talking to them. By staff raising their voices this could lead to perceptions of threat or misunderstanding thus escalating the situation.

\section{Calming strategies (blue zone)}

After an episode of CB both the person and caregiver are likely to maintain a heightened level of arousal. Any knowledge about what actually triggered the behaviour is useful, which may then require a move to a different setting to allow the person to calm down. It is often best not to place any unwarranted extra demands on the person during this time, and so normal rules about use of dining room, usual bedtime, etc., may need to be temporarily relaxed. As previously mentioned, the person may easily be re-triggered into another distressed episode and it is important that staff offer support in these post-event periods.

Accurate reporting of all incidents can be used to identify risks and to develop prevention strategies (Stout, 2008). To understand and learn from such incidents, it is useful to record the incident using some form of time-series monitoring chart, such as an $\mathrm{ABC}$ chart. Following a serious incident there should be a post-incident review. This should take place as soon after the incident as possible, while accounting for any trauma, both physical and mental, that those involved may have experienced (NHS Counter Fraud and Security Management Service, 2003). The NHS Counter Fraud and Security Management Service (2003) identified several questions that should be asked during the review, including: What can be learned? What can be done to avoid repeating mistakes? How can what is and is not working be assessed? What are the implications of what happened? Are policy and system revisions required?

\section{Implications of the arousal cycle and traffic light analogies}

The traffic light system assists in the organising of training and the determining of the clinical pathway for CBs. For example, it helped in the development of the training programme CAIT 


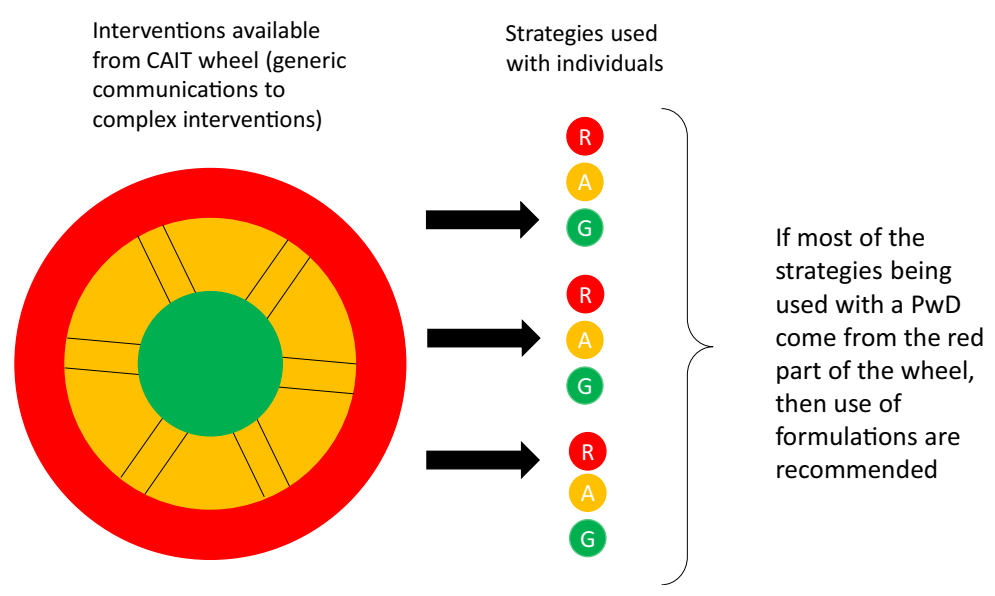

Figure 3. CAIT wheel guidance for the use of formulations and care planning.

(James and Gibbons, 2019), which emphasises the importance of caregiver communication skills. This was developed to reduce levels of emotional and behavioural stress and distress in people with dementia. Such agitation can occur because of poor communication and often results in the prescribing of tranquilising and sedating medications (James and Jackman, 2017). Our clinical work shows that the strategies we recommend in this training have a double impact: (i) they reduce the likely occurrence of distress and problematic behaviours, and (ii) they help de-escalate situations in which the $\mathrm{PwD}$ has become distressed or agitated. CAIT has been used in a three-year pilot project with 30 care homes in Sunderland, UK. A more controlled assessment of its impact is currently underway (James and Gibbons, 2019).

CAIT is built on basic communication principles and is represented visually in the form of a wheel, with the hubs, spokes and rim (James, 2015; James et al., 2017). Aspects of the 'wheel of communication' are discussed in four training modules, which are reviewed below (Fig. 3).

\section{Core general communication - inner hub of wheel/module 1}

The training in this module is built around the central skill of good, basic, respectful communication. Such skills are similar to those taught to retail staff and law enforcement officers around customer care. We believe that ensuring caregivers are using empathic, considerate communication enhances levels of wellbeing and reduces the need for some of the more advanced strategies.

\section{Dementia-specific communication - outer hub of wheel/module 2}

This module examines the adaptations required to communicate with people with dementia, taking account of memory and sensory changes. The nature of diagnosis is discussed, together with the skills needed in day-to-day interactions, such as: body language, appropriate pacing, use of personal space, types of questions to use/avoid, persuasion and negotiation skills.

\section{Activities of daily living (ADL) - wheel spokes/module 3}

Caregivers are often supporting people to undertake personal tasks, including hygiene activities. Owing to the intimate nature and potential invasiveness surrounding ADLs, these activities are often triggers to agitation; as such these activities may be viewed as 'predictable' triggers. 
To minimise distress and agitation, we coach the hands-on carer skills required to manage intimate care tasks in a sensitive, dignified and empathic manner. In this section de-escalations skills are taught (e.g. distraction, re-direction, therapeutic lies), because the close interactions during the ADLs are frequently triggers to CBs.

\section{Complex and formulation-led approaches - wheel rim/module 4}

Advanced skills are taught in this module with the aim of managing highly problematic emotions and behaviours. This may involve the use of specialist assessment and management techniques used in keeping everyone safe. In this section the enhanced care plans, the role of hand holds and other restraining techniques are demonstrated and discussed.

In relation to the traffic light model, the first two modules of the CAIT wheel (inner and outer hub) address 'green' techniques because they are promoting levels of wellbeing. Module 3 (ADLs) focuses on many of the amber strategies. Module 4 provides 'red' techniques, developing skills in obtaining a comprehensive understanding and keeping people safe (see Fig. 3). It is relevant to note that module 4 attempts to incorporate all the various features of the previous modules in an integrated manner. This is the reason that module 4 is associated with training and supervision of formulation skills.

In an earlier section we spoke about the importance of formulations and their relevance to the management of CB. However, our experience suggests that formulations are not routinely used in many settings because of a lack of both a perceived need and also the resources available within the settings (including limited time and skills in producing them). As such, the use of formulations usually needs to be rationalised and mainly used in those cases where it is felt to be a priority. It is believed that the CAIT wheel can provide a useful clinical template to guide the prioritisation of the use of formulations (Fig. 3). This template would suggest that they should be used in those circumstances where clinicians are routinely working using skills taught in the red or amber modules with respect to a person with dementia (i.e. if a clinician is frequently using red and amber strategies during someone's care).

The resulting care plans are also usefully structured around the traffic light framework (Table 2), with the care plans providing details of: how to maintain someone's wellbeing (green); likely triggers and de-escalation strategies (amber); and how to work with the individual when they are extremely distressed (red).

We perceive the addition of the arousal cycle and the traffic light analogy to be helpful additions to the toolkit for the management of CB. It is important to note, however, that for the sake of simplicity we have presented the movement between the phases in a rather linear progressive manner. In reality, the movement between phases within the cycle are likely to be complex and dynamic. Hence tracking where the person with dementia is situated in the cycle at any point in time will require careful monitoring and empathic understanding on the part of the caregiver.

This article has provided a broad sweep of models and frameworks from within and outside of the field of dementia. Some of the positive behavioural elements are already being used by dementia teams across the UK (Surrey and Borders Partnership NHS Foundation Trust, and Cumbria, Northumberland, Tyne and Wear NHS Foundation Trust; Sells and Shirley, 2010). However, up to this point there has not been an attempt to integrate the models with the practical protocols we have mentioned in this article. Hence, owing to the novelty of our work, its value and efficacy should be questioned, debated and road-tested. In addition, the costs and benefits associated with these approaches need to be assessed. 
Table 2. Example of a traffic-light informed care plan

\begin{tabular}{|c|c|}
\hline Strategies & Details of colour-coded care plan activities for Mrs 'Mary' Jones \\
\hline \multirow[t]{5}{*}{ Green } & $\begin{array}{l}\text { Talk into Mary's left ear and try using communication cards/writing to help express her } \\
\text { needs/wishes (as she struggles to hear) }\end{array}$ \\
\hline & $\begin{array}{l}\text { Ask Mary to clean the bedroom, dust the shelves or generally help out. She likes things to be } \\
\text { tidy and will help with laying tables/folding laundry }\end{array}$ \\
\hline & $\begin{array}{l}\text { Play Mary's favourite music (Abba, Roy Orbison) and TV shows (Coronation Street and On } \\
\text { the Buses). She loves a Cornetto for a treat! }\end{array}$ \\
\hline & $\begin{array}{l}\text { Read a book, magazine or newspaper with Mary. She likes to read out the headlines and } \\
\text { will discuss current affairs (Daily Star) }\end{array}$ \\
\hline & $\begin{array}{l}\text { Ask Mary about the cruise she went on (to Norway) and her pet dog, Barney (golden } \\
\text { retriever) }\end{array}$ \\
\hline \multirow[t]{5}{*}{ Amber } & $\begin{array}{l}\text { Know Mary's triggers: boredom can be a big trigger for her distress, particularly in the long } \\
\text { period after lunch. She also does not like to sit near Mrs } Q\end{array}$ \\
\hline & $\begin{array}{l}\text { Change Mary's environment: take her to the garden, quiet lounge or reception desk. Try to } \\
\text { engage Mary in vigorous cleaning (such as polishing or washing windows) to distract her } \\
\text { and cope with emotions }\end{array}$ \\
\hline & $\begin{array}{l}\text { Bring in preferred staff members (carer Suzy or Mark the chef). If this does not work, } \\
\text { introduce an authority figure (Home Manager or Nurse in Charge) as Mary is more likely } \\
\text { to accept medication/ADLs from them }\end{array}$ \\
\hline & $\begin{array}{l}\text { Ask for help/advice: If you need to stop Mary doing something, avoid banned words like } \\
\text { 'no', 'stop', 'don't' etc. Instead, say 'I'm so glad I've seen you - might you be able to lend a } \\
\text { hand with the laundry please?' }\end{array}$ \\
\hline & Show Mary you are respecting her by calling her 'Mrs Jones' \\
\hline \multirow[t]{4}{*}{ Red } & $\begin{array}{l}\text { Back off and make things safe: leave Mary alone for a few minutes, particularly if she starts } \\
\text { to throw things, pulls hair or screams. Ensure other residents are moved away for their } \\
\text { safety }\end{array}$ \\
\hline & $\begin{array}{l}\text { Stay calm: Show Mary that you can cope and want to help to her. Use simple, factual } \\
\text { statements, and only one staff member should talk at a time. Use open, relaxed body } \\
\text { language to show you are not a threat and want to help }\end{array}$ \\
\hline & $\begin{array}{l}\text { She dislikes putting on her shoes, and tends to pull your hair when you try to assist. To } \\
\text { prevent this happening, ask her to hold the therapeutic doll in order to keep her hands } \\
\text { occupied - (NB she loves the doll and so is unlikely to use it as a weapon). }\end{array}$ \\
\hline & $\begin{array}{l}\text { When she asks to see her deceased husband, employ the therapeutic lie described in detail in } \\
\text { her notes. She should be informed that he is away on his fortnightly visit to see his infirmed } \\
\text { younger brother. This answer is currently the best at calming her down, and is fully consistent } \\
\text { with her life-story }\end{array}$ \\
\hline
\end{tabular}

\section{Conclusion}

NICE (2018) suggests the use of non-pharmacological treatments as first-line approaches, but does not provide any specific guidance on what approaches to use for CB. The present paper provides some models that will be unfamiliar to many people working in dementia care and that link directly to management strategies. The novel approaches have been integrated with more familiar frameworks such as the 'unmet needs' and formulation-led methodologies. We believe the fusion of the old and new provides us with practical treatment protocols that can be readily incorporated into training programmes. We are currently unable to support the suggestions made in the article with an evidence base and therefore must be cautious in recommending them. Nevertheless, there is tentative support for some of our perspectives from the training programme CAIT, as well as from longstanding effective use of some of these concepts in clinical settings within two NHS Trusts (Sells and Shirley, 2010).

This paper is part of a programme of work which is attempting to clarify the mechanisms of change regarding the treatment of CB. In the ideal case scenario, we would want caregivers who are supporting people with dementia to be able to fully articulate the approaches they are using to meet people's needs and de-escalate difficult situations. We believe such articulation 
will empower caregivers, and allow them to pass on their skills to junior members of their clinical team. It is suggested that the very practical nature of this paper, employing useful paradigms from other fields, is a helpful step in clarifying 'who should do what, when and how'.

Acknowledgements. The authors would like to thank Dr Joanna Marshall, Dr Paula Maisey and Barbara Medea for the intellectual contributions to this article.

Financial support. The research received no grant from any funding agency, commercial or not-for-profit organisations.

Conflicts of interest. The authors have no conflicts of interest with respect to this publication.

Ethical statement. No ethical approval was required for the preparation of this article as it is a practice article and no participants were used.

Key practice points

(1) A structural analysis of disruptive behaviours allows for better targeted interventions, which can be successfully care planned using a traffic light analogy.

(2) Challenging behaviours are dealt with across a number of specialities; a greater sharing of knowledge and skills across the specialities would be helpful. In this article we have demonstrated the way in which Positive Behavioural Support concepts have been useful in producing guidelines in dementia care.

(3) Care plans can be improved by conceptualising the structural elements of the $\mathrm{CB}$ in addition to the biopsychosocial features of the person with dementia.

(4) Effective communication is the bedrock of all successful CB interventions. Poor communication can lead to disruptions because people's 'needs' for security, respect control may be undermined and go 'unmet'. There are a number of communication programmes (e.g. CAIT) designed to help improve caregivers' interactions.

(5) The practical nature of the paper provides caregivers with insights into some of the strategies they are using intuitively. Such insights will help them to be able to articulate their skills, which they can then pass on to colleagues and enhance the level of care provided to people with dementia.

\section{Further reading}

Ballard, C., Corbett, A., Orrell, M. et al. (2018). Impact of person-centred care training and person-centred activities on quality of life, agitation, and antipsychotic use in people living with dementia: a cluster randomised control trail. PLoS Medicine, 15, e1002500

Moniz-Cook, E., Roberts-Stride, K., James, I. et al. (2012). Functional analysis-based interventions in challenging behaviour in dementia. Cochrane Database, 1, CD006929. doi: 10.1002/14651858.CD006929

\section{References}

Algase, D. L., Beck, C., Kolanowski, A., Whall, A., Berebt, S., Richards, K. et al. (1996). Need-driven dementia compromised behavior: An alternative view of disruptive behavior. American Journal of Alzheimer's Disease, 11, 12-19.

Ballard, C., Orrell, M., YongZhong, S., Moniz-Cook, E., Stafford, J., Whittaker, R., Woods, B., Corbett, A., Garrod, L., Khan, Z., Woodward-Carlton, B., Wenborn, J., \& Fossey, J. (2016). Impact of antipsychotic review and nonpharmacological intervention on antipsychotic use, neuropsychiatric symptoms, and mortality in people with dementia living in nursing homes: a factorial cluster-randomized controlled trial by the Well-Being and Health for People with Dementia (WHELD) Program. American Journal of Psychiatry, 173, 252-262.

Banerjee, S. (2009). The Use of Antipsychotic Medication for People with Dementia: Time for Action. London, UK: Department of Health.

Brechin, D., Murphy, G., James, I. A. \& Codner, J. (2013). Alternatives to Antipsychotic Medication: Psychological Approaches in Managing Psychological and Behavioural Distress in People with Dementia. Division of Clinical Psychology. BPS: Leicester.

Brooker, D. \& Latham, I. (2015). Person-Centred Dementia Care: Making Services Better With VIPS Framework. London, UK: Jessica Kingsley.

Bunn, F. \& Handley, M. (2019). Reducing agitation in care home residents with dementia. The Lancet Online, 6, 274. http:// dx.doi.org/10.1016/S2215-0366(19)30080-X 
Cerejeira, J., Lagarto, L. \& Mukaetova-Ladinska, E. (2012). Behavioral and psychological symptoms of dementia. Frontiers in Neurology, 3, 73. doi: 10.3389/fneur.2012.00073

Cohen-Mansfield, J. (2000). Use of patient characteristics to determine non-pharmacological interventions for behavioural and psychological symptoms of dementia. International Psychogeriatrics, 12, 373-380.

Cunningham, C., Macfarlane, S., \& Brodaty, H. (2019). Language paradigms when behaviour changes with dementia: \#BanBPSD (Editorial). International Journal of Geriatric Psychiatry, 34, 1109-1113.

Gilbert, P. (2014). The origins and nature of compassion focused therapy. British Journal of Clinical Psychology, 53, 6-41.

Hallet, N. (2018). Preventing and managing challenging behaviour. Nursing Standard, 32, 51-63. doi: 10.7748/ns.2018.e10969

Holle, D., Halek, M., Holle, B. and Pinkert, C. (2016). Individualized formulation-led interventions for analyzing and managing challenging behavior of people with dementia - an integrative review, Aging and Mental Health, 21, 1229-1247. doi: 10.1080/13607863.2016.1247429

Howarth, A., Crooks, M. and Sells, D. (2017). The use of physical restraint to deliver essential personal care to incapacitated older adults with dementia. In I. James \& L. Jackman (eds), Understanding Behaviour in Dementia that Challenges (2nd edn). London, UK: Jessica Kingsley Press.

Howarth, A. \& Sells, D. (2014). The Forced Care Framework: guidance for staff. Journal of Dementia Care, 22, 30-34.

HSJ (2020). Watch out for the trend: mental health matters (Rebecca Thomas). Health Service Journal (22 June 2020).

James, I. A. (2015). The use of CBT in dementia care: a rationale for Communication and Interaction Therapy (CAIT) and therapeutic lies. The Cognitive Behaviour Therapist, 8, e10.

James, I. A., \& Gibbons, L. (2019). Communication Skills for Effective Dementia Care: A Practical Guide to CAIT. London, UK: Jessica Kingsley Press.

James, I. A., \& Jackman, L. (2017). Understanding Behaviour in Dementia that Challenges (2nd edn). London, UK: Jessica Kingsley Press.

James, I., Marshall, J. \& Thwaites, S. (2017). Improving communication skills in dementia care: utilising the British Psychological Society's stepped-care model for treatment of behaviour that challenges. Psychology of Older People: The FPOP Bulletin, 137, 36-41.

James, I. A., Medea, B., \& Reichelt, K. (in press). De-escalation strategies in the management of challenging behaviour in dementia: creating a competent and articulate workforce. Psychology of Older People: The FPOP Bulletin.

James, I. A., \& Moniz-Cook, E. (2018). Evidence briefing: behaviour that challenges in dementia. British Psychological Society. www.bps.org.uk

James, I. A., \& Reichelt, K. (2019). Understanding people's needs: the 8-needs framework for the treatment of behaviours that challenge. Psychology of Older People: The FPOP Bulletin, 147, 24-30.

Kaplan, S., \& Wheeler E. (1983). Survival skills for working with potentially violent clients. Social Casework, 64, 339-346.

Kitwood, T. (1997). Dementia Reconsidered: The Person Comes First. Buckingham: Open University Press.

Livingston, G., Kelly, L., Lewis-Holmes, E., Baio, G., Morris, S., Patel, N., Omar, R. Z., Katona, C., \& Cooper C. (2014). A systematic review of the clinical effectiveness and cost-effectiveness of sensory, psychological and behavioural interventions for managing agitation in older adults with dementia. Health Technology Assessment, 18, 1-226. doi: 10.3310/hta18390.

Macaulay, S. (2015). B is for BANGS: care partnering, challenging and solutions, tips and tools and skills (http:// myalzheimersstory.com/2015/06/18/b-is-for-breathe-in-bangs/).

McCallion, P., Toseland, R. W., Lacey, D., \& Banks, S. (1999). Educating nursing assistants to communicate more effectively with nursing home residents with dementia. Gerontologist, 39, 546-558. doi: 10.1093/geront/39.5.546

Moniz-Cook, E., Roberts-Stride, K., James, I. et al. (2012). Functional analysis-based interventions in challenging behaviour in dementia. Cochrane Database, 1, CD006929. doi: 10.1002/14651858.CD006929

NHS Protect (2017). Guidance on the Prevention and Management of Clinically Related Challenging Behaviour in NHS Settings: Meeting Needs and Reducing Distress. http://www.reducingdistress.co.uk/reducingdistress/wpcontent/uploads/ 2014/02/Meeting_needs_and_reducing_distress.pdf (accessed 31 January 2018.)

NHS Counter Fraud and Security Management Service (2003). Conflict Resolution Training: Implementing the National Syllabus. NHS, London.

NICE (2018). Dementia: assessment, management and support for people living with dementia and their carers. Guidance/ng97.

Rapaport, P., Livingston, G., Murray, J., Mulla, A., \& Cooper, C. (2017). Systematic review of the effective components of psychological interventions delivered by care home staff to people with dementia. BMJ Open, 7, e014177.

Sells, D., \& Shirley, L. (2010). Person-centred risk management: the traffic light approach. Journal of Dementia Care, 18, 21-23.

Stout, N. (2008). The public health approach to occupational injury research: from surveillance to prevention. Safety Science, $46,230-233$.

Cite this article: James IA, Reichelt K, Moniz-Cook E, and Lee K. Challenging behaviour in dementia care: a novel framework for translating knowledge to practice. The Cognitive Behaviour Therapist. https://doi.org/10.1017/S1754470X20000434 